\title{
Traversées Atlantiques et Poétique Romanesque chez Alejo Carpentier: Voyages, échanges, transferts
}

\section{Daniel-Henri Pageaux \\ Sorbonne Nouvelle/Paris III}

Résumé: Il existe un trajet fondateur dans le roman carpentiérien: la traversée de l'Atlantique. Le passage du vieux au nouveau monde dessine une dynamique première: celle du dialogue des cultures, de l'interculturalité qui est au cœur de la réflexion et de l'imaginaire du romancier. On étudiera le personnel romanesque (féminin en particulier) qui, dans leurs traversées atlantiques, opèrent une métamorphose, puis les personnages noirs qui ont été les acteurs symboliques de ces traversées. Une attention particulière sera accordée aux idées qui, elles aussi, traversent l'Atlantique, en particulier le baroque, qui va donner au continent américain une identité nouvelle et de nouveaux moyens d'exprimer son universalité.

Mots-clés: Femmes, Homme noir, Réel merveilleux, Baroque

\begin{abstract}
There is a founding trajectory in the novels of A. Carpentier: the crossing of the Atlantic. The journey from the old to the new world frames a primordial dynamic: a dialogue of cultures, the interculturalism at the center of the novelist's reflection and imaginary. This paper is an analysis of the fictional characters, particularly the female ones, who, on their Atlantic crossings, undergo a metamorphosis; it focuses, as well, on the black characters who are the protagonists of these crossings. Special attention is given to the ideas which, too, cross the Atlantic, in particular the Baroque, which offered the American continent a new identity and new means of expressing its universality.
\end{abstract}

Keywords: Woman, black man, marvelous realism, Baroque 
Il existe un trajet fondateur dans l'univers romanesque d'Alejo Carpentier: la traversée de l'Atlantique. Le passage du vieux au nouveau monde (ou l'inverse) dessine une dynamique première, essentielle: celle du dialogue des cultures, de l'interculturalité, au cœur de la réflexion et de l'imaginaire du romancier. Du point de vue de l'invention romanesque, des personnages passent d'Europe en Amérique. Ont-ils comme ancêtre ou modèle Colomb, le "découvreur"? Oui, sans doute et le dernier roman de Carpentier, $L a$ Harpe et l'Ombre/El Harpa y la Sombra qui prend comme argument le projet de canonisation du "découvreur" au XIXème siècle, témoigne de l'intérêt teinté d'ironie que le romancier a porté à celui qui a fait dialoguer ancien et nouveau monde.

Il faut compter aussi avec la première et dramatique conséquence de la découverte des "Indes": la traite, l'arrivée massive d'esclaves venus d'Afrique occidentale. Ce sont des figures romanesques inspirées d'un phénomène transcontinental qui concerne au premier chef l'espace caraïbe. Il y a donc un problème d'articulation entre deux séries d'espaces: d'une part, l'ancien et le nouveau monde, d'autre part, l'Afrique et les Caraïbes. Si le personnage noir, esclave ou descendant d'esclave, marque durablement les romans de Carpentier et révèle un profond investissement symbolique - l'homme noir idéalisé et porteur d'idées salvatrices, voire révolutionnaires - il convient d'étudier avant tout, en suivant une chronologie à la fois biographique et poétique, trois personnages féminins qui traversent l'Atlantique. Venues d'Europe, elles vont connaître une expérience "américaine", centrée, là encore, sur l'aire caraïbe, Haïti ou Cuba. Mais on peut parler d'une transformation profonde de leur être qui n'aurait pas été possible sans l'abandon de l'Europe et l'embarquement pour le Nouveau Monde.

Ce ne sont pas seulement les êtres qui voyagent. Les idées passent par-dessus l'Atlantique, de même que les formes et les mots. Le baroque, venu d'Europe, devient, pour Carpentier, l'esthétique qui définit une identité américaine, tandis qu'il rappelle en exergue à son roman, El Siglo de las Luces/Le Siècle des Lumières que "Les paroles ne tombent pas dans le vide" (Carpentier 2005: 17). Entendons: l'échec n'est jamais négatif et l'échec fait progresser. Au-delà des êtres et des idées, une poétique s'élabore, fondée sur l'affirmation répétée de la "supériorité" du nouveau monde sur l’ancien. Mais cette supériorité a pour 
conséquence d'intégrer, enfin, le "continent" américain dans un ensemble plus vaste que Carpentier nomme "l'universel".

Trois personnages féminins deviennent les héroïnes non seulement d'une traversée atlantique, mais d'une authentique métamorphose: Pauline Bonaparte dans El Reino de este mundo/ Le Royaume de ce Monde, Sofía/Sophie dans Le Siècle des Lumières et Vera dans La Consagración de la Primavera/ La Danse Sacrale. Ces trois femmes incarnent un dynamisme vital, un processus physique et moral en continuelle transformation. Êtres humains, pris dans sa singularité, les femmes sont des voyageuses, en transit, entre deux mondes. Le déplacement, le passage d'une culture à une autre sont chez elles une composante majeure de leur personnalité. Mais elles illustrent aussi le phénomène qui inaugure, pour le "souscontinent", l'ouverture sur la modernité, el modernismo que Max Henríquez Ureña, historien de ce mouvement, a nommé, de façon imagée, mais symbolique, "le retour des galions"/el retorno de los galeones. Le voyage atlantique se fait à présent dans les deux sens, il y a échanges et l'Amérique a quelque chose à dire et à apporter à l'Europe. C'est aussi la prise de conscience à partir de laquelle Carpentier invente ses romans.

Pauline est un personnage historique qui a fasciné Carpentier en ce qu'il lui a permis très tôt, comme il le rappelle dans Tientos y Diferencias/Essais et Différences (Carpentier 1976: 94), de découvrir "des synchronismes possibles, américains, récurrents, au-delà du temps, mettant en relation ceci avec cela, hier avec aujourd'hui". Retenons ces mots de présentation que nous retrouverons, sur un autre plan, plus général, à la fin de cet article. Elle a été son "guide" (lazarillo y guía). Initiatrice, elle a montré, dans son aller-retour atlantique, de nouvelles possibilités d'écriture. A l'expérience existentielle du personnage correspond l'expérience poétique du romancier qui parlera de "révélation"/revelación. Nous reviendrons, là aussi en fin de parcours, sur ce terme, essentiel pour la poétique du romancier.

Pauline Bonaparte fait une apparition fugitive dans le texte qui servira de préface au 
Royaume de ce Monde ("la théorie" du réel merveilleux). Dans ce même roman, elle sera un personnage épisodique, elle aura, dans l'esprit et l'imaginaire du romancier (comme celui-ci l'a commenté à plusieurs reprises), une valeur d'exemple et de modèle. Pauline Bonaparte, sœur du Premier Consul, Napoléon Bonaparte, épouse du Général Leclerc, apparaît dans la deuxième partie du roman. Celle-ci s'ouvre sur une citation des Mémoires de la Duchesse d'Abrantès, en français, qui évoque le prochain départ pour les îles de Pauline en ces termes: "J'achevais mon discours en lui disant qu'elle serait bien jolie mise en créole" (Carpentier 1980: 56). ${ }^{1}$ Pour la duchesse, le mot "créole" renvoie au costume folklorique, au déguisement vaguement érotique et exotique de la "doudou" des "isles". Mais le mot peut aussi signifier, dans un contexte linguistique et culturel hispanique, celui du romancier, la femme, qu'elle soit ou non d'origine espagnole ou européenne, née et élevée aux Indes (du verbe espagnol criar). Cette mise en scène "créole" annoncée dessine, de fait, la trajectoire vitale du personnage.

Au cours du voyage d'aller à Haïti (le Général Leclerc vient pour rétablir l'esclavage), Pauline note un changement atmosphérique au moment d'aborder les Açores: elle est aux portes d'un nouveau monde et la mer "change" (idem: 90). ${ }^{2}$ Cette mer n'est plus celle du vieux monde, mais un espace qui annonce de nouveaux horizons. On notera, dans le texte espagnol, l'effet d'écho ou plutôt l'effet de sens entre "nouveau monde" et le gérondif "renovando" ("en train de se rénover"). Mais Pauline est encore prisonnière de mirages exotiques et sentimentaux de la fin du siècle des Lumières, en particulier ceux du roman à succès Paul et Virginie. Après l'épreuve de l'épidémie de vomito negro qui coûtera la vie à son mari, son exil à l'île de Tortue va la transformer en véritable "créole" hispanique. L'image de la Corse, son île natale, se brouille pour se changer en une île des Caraïbes, selon un traitement poétique de l'espace, constant dans le monde romanesque de Carpentier: qu'il s'agisse de terre ou de mer, l'espace auquel est dévolue une charge poétique, évolue, se dédouble. Pauline retournera en Europe avec le cadavre de son mari et ses "déguisements fanés de créole" (idem: 99), mais aussi transformée, murie, emportant une amulette de Papa Legba, cadeau du nègre Soliman qui avait été son guérisseur pendant l'épidémie. On 
retrouvera dans la IVème partie du roman, en un parallélisme oppositionnel significatif, Soliman qui sombre dans la folie et Pauline, statufiée par Canova.

Carpentier, mélomane et musicologue averti, a défini à plusieurs reprises El Siglo de las Luces comme une "sonate à trois voix". Victor Hugues qui "fait" la révolution au nom de la Convention, mais qui va aussi la défaire, la renier, toujours au nom d'une politique et qui apparaît, pour reprendre les mots du romancier, comme une hypostase de Robespierre (Victor Hugues ou le "Robespierre des isles"). Face à lui, le jeune Esteban, conçu dès le début du roman sur le modèle christique (il est comparé, lors de ses crises d'asthme, à un Christ souffrant du peintre Berruguete), jeune homme sensible, idéaliste: il adhère à l'idée vague d'une révolution totale et sera évidemment déçu. Enfin, Sofía, amoureuse de Victor Hugues, et qui va, à sa manière, se libérer, en décidant, après son veuvage, de rejoindre son amant et ensuite de le quitter, quand elle sera parvenue au bout de la déception. Tout au long du roman, Sofía répète: "Il faut faire quelque chose". On peut dire qu'avec cette formule elle exprime, de façon laconique, l'idéalisme foncier qui l'anime. Elle la prononcera encore à la fin du roman, à Madrid, en plein soulèvement du Dos de Mayo, avant de disparaître, avec son cousin Esteban, dans le tourbillon de l'émeute madrilène. Ce couple ne meurt pas à la fin de cette sorte d'épopée qui fait passer d'un ordre ancien à un monde nouveau; notons qu'il y a deux figurations de la révolution dans ce roman: au sens politique du terme il faut ajouter l'image de la révolution cosmique, à la fois morale et poétique. C'est elle qui emporte Sofía et Esteban: ils s'en vont dans l'espace en mouvement; ils sont devenus des "absents" (Carpentier 2005: 462). ${ }^{3}$

El Siglo de las Luces est le roman de la Révolution manquée, parce qu'elle est la Révolution qui n'a pu être exportée: le roman - rappelons-le - s'ouvre sur l'image de la "Machine", c'est-à-dire de la guillotine, qui vogue sur les eaux de l'Atlantique en compagnie du "révolutionnaire" Victor Hugues. Mais, si l'on reprend cette première séquence du roman, sorte de poème en prose, détaché du contexte historique, chronologique, on notera que le bateau qui transporte une certaine idée de la révolution est saisi au moment même où il va entrer dans la mer des Sargasses: autant dire au moment où le bateau et avec lui la Révolution vont, symboliquement, sinon s'enliser, du moins ne plus progresser. Il faudra 
attendre une révolution née au sein même de l'espace américain - le soulèvement emmené par Fidel Castro - pour que l'idéal de la révolution puisse réussir, le "Grand Projet", mots employés par Vera dans La Danse Sacrale.

Méfiante à l'égard du processus révolutionnaire - elle est une Russe émigrée - Vera ne peut pourtant s'empêcher d'être aux côtés des républicains espagnols et d'admirer les soldats de Castro. Mieux: elle compare, premier pas vers l'adhésion à la révolution, les barbudos aux chevelus de Valmy. Vera qui a résisté à la révolution russe, part à la recherche de "quelque chose"/algo, en italique dans le texte. Ce faisant, elle rejoint bien évidemment Sofía. Bien plus, Vera se souvient du précepte de Gogol: "Ne fuis pas le monde dans lequel il t'a été donné de vivre", ce que l'on peut tenir pour la version "russe" du "royaume de ce monde" /reino de este mundo caribéen, tel que le déclame à tous les vents l'esclave Ti Noel à la fin du roman éponyme.

Deux épigraphes éclairent le sens de l'action humaine, telle qu'elle se dessine à partir du couple de personnages. Notons au passage, comme dans le cas de Sofía/Esteban, l'interdépendance des deux personnages masculin et féminin. Au chapitre VIII qu'on appellera le chapitre d'Enrique, une phrase de Herman Melville mise en épigraphe: “Quand achèverons-nous de nous accomplir? Tant qu'il nous restera quelque chose à faire nous n'avons rien fait" (Carpentier 1978: 477; nous soulignons) montre bien comment l'idée de l'amélioration du monde passe aussi par une vision intérieure, un engagement personnel, ce que l'on peut appeler une révolution intérieure. Au chapitre IX, le chapitre de Vera, une citation du Second Faust de Goethe: "Celui-là seul mérite la liberté et la vie qui doit chaque jour les conquérir" (idem: 519) met encore plus l'accent sur le contrat exigeant que l'homme doit passer avec lui-même non seulement pour lutter, mais pour se dépasser de façon quotidienne et continue.

Telle est donc la face double de cette révolution selon Carpentier: enracinée dans la conjoncture historique, attentive à la condition matérielle et spirituelle de l'homme, elle aspire aussi à une transformation radicale de l'homme. A la spirale de l'histoire (l'histoire semble se répéter, mais en fait elle avance), correspond le lent cheminement de tout homme dans "le royaume de ce monde". C'est bien le sens métaphorique (un mot clé de la 
poétique de Carpentier, comme nous le verrons) qu'il faut donner au Chat de Lewis Carroll qui revient soudain à la mémoire de Vera et qui lui rappelle: "Vous pouvez être sûre d'arriver quelque part si seulement vous marchez assez longtemps".4

Au-delà des destins individuels, La Danse Sacrale marque le dernier temps de la quête de la Révolution, dans et par le roman. Or, la Révolution, née en Europe, sort finalement victorieuse dans le Nouveau Monde. Le fil conducteur est apparemment double: d'un côté, Vera, sinon hostile, du moins réservée à l'égard de la révolution, quelque peu sœur de Sofía; de l'autre, Enrique, double d'Esteban à certains égards et donc aussi du romancier. Il s'est engagé dans la guerre d'Espagne, mais il va, comme Vera, être pris dans un processus révolutionnaire dans lequel le Noir Calixto a aussi son rôle à jouer. Calixto croit à la révolution parce qu'il a tout à gagner: en cela il rappelle - on va le voir - la conduite et les opinions du noir Filomeno dans Concierto Barroco.

L'homme noir, un certain homme noir, traverse de part en part l'œuvre romanesque d'Alejo Carpentier (1904-1980). Citons les principaux jalons depuis ¡Ecué-Yamba-Ó! (1933)/Ekoué-Yamba-Ó, premier roman longtemps renié, jusqu'à La Danse Sacrale (1978), en passant par Le Royaume de ce Monde (1949) et Concierto Barroco/Concert Baroque (1974). Nous centrerons notre attention sur quatre personnages aux traits bien incertains, même si le terme est peu satisfaisant. Leur trajectoire de vie - comme dans le cas des personnages féminins, propose, de diverses manières, un dépassement de leur condition sociale et raciale, dans un cadre essentiellement cubain, caraïbe, pour accéder à une dimension morale, voire à une fonction symbolique qui font de ces infimes représentants du peuple ou du prolétariat noir des figures qu'on peut à bon droit tenir pour mythiques.

¡Ecué-Yamba-Ó!, qu’on traduira par “Dieu soit loué”, est une œuvre de jeunesse. Ecrit ou commencé en prison, en 1927, alors que Carpentier est opposant à la dictature de Gerardo Machado, le roman est repris, remanié en France et publié en Espagne en 1933, sans grand succès. La sortie d'une édition pirate amène l'écrivain à republier le roman, 
assorti d'une préface explicative plutôt sévère. ${ }^{5}$ Le roman est l'histoire, courte au demeurant, de Menegildo Cué, depuis sa naissance jusqu'à son assassinat par une bande rivale. Né au sein d'une pauvre famille d'anciens esclaves, employés d'une usine à sucre, victimes de la crise économique, il commence par aider son père dans le travail de la terre. Au sortir de l'adolescence, il tombe amoureux de Longina, d'origine haïtienne, mariée. Il poignarde le mari et connaît la prison. Libéré, il est initié par son cousin Antonio et devient membre d'une confrérie ñañiga. Le mot et l'institution ("el ñañiguismo") méritent un long paragraphe explicatif dans le glossaire établi par Carpentier: il s'agit à la fois "d'associations secrètes de protection mutuelle" (Carpentier 1988: 225) et "d'une sorte de francmaçonnerie populaire" (idem: 225). Lors d'un affrontement entre confréries rivales, Menegildo trouve la mort. Quelques mois plus tard, Longina, qui s'est réfugiée auprès de Salomé, mère de Menegildo, donne naissance à un garçon qu'on appelle Menegildo. Et, pour le préserver des maléfices, "une bougie de sainte Thérèse brûlait en son honneur devant la très chrétienne statue de saint Lazare-Babayú-Ayé" (idem: 211). C'est sur ce dernier exemple de syncrétisme religieux (infime expression d'un transfert culturel, également), caractéristique de la santería cubaine, que se termine le roman. Mais la fin renvoie à la première "initiation" (titre du chapitre 4 (idem: 35) quand Salomé allume, "pour préserver le nourrisson de nouveaux dangers [...] allume "un petit cierge de Sainte Thérèse devant la statue de saint Lazare qui présidait l'autel" (idem: 37). La vie de Menegildo qui se présentait comme une succession de courtes scènes obéit en fait à un principe cyclique.

Le roman accorde une large place aux coutumes, fêtes, chants, pratiques religieuses: en cela il est caractéristique des années d'apprentissage de Carpentier, marquées par un très fort intérêt pour la culture afro-cubaine. La rédaction ou les remaniements du roman sont contemporains d'articles où Carpentier présente les "sorciers" de Cuba, la musique cubaine qui fait fureur à Paris où il va vivre pendant une décennie jusqu'en 1939. Plusieurs chants cités dans le roman seront pris de l'ouvrage La Música en Cuba (1946), point d'aboutissement de recherches menées par Carpentier, à la suite des travaux du grand prédécesseur, l'ethnomusicologue Fernando Ortiz (idem: 45, 81, 104, 117). 
La découverte d'Haïti en 1943 et, plus encore, le plaidoyer pour un "réel merveilleux", un article publié le 8 avril 1948 dans le journal El Nacional, repris l'année suivante comme préface au roman Le Royaume de ce Monde, marquent la fin de l'afrocubanisme de Carpentier, même s'il témoigne, au long de sa vie, un vif intérêt pour la musique de son île natale. Le "réel merveilleux" est la notion esthétique que Carpentier invente contre le surréalisme parisien et dont il s'est écarté, en particulier sous l'influence de son ami Robert Desnos. Il va servir à l'expression d'une identité américaine, c'est-à-dire un moyen d'affirmer la supériorité de l'Amérique face à une Europe trop intellectualisée et présentée comme épuisée (Carpentier 1983: 342-349).

La vie de Ti Noel (Carpentier a trouvé ce nom, comme bien d'autres détails, dans la Description Topographique de la Partie Française de l'Île de Saint-Domingue de Moreau de Saint Méry) sert de fil conducteur au roman, des dernières années de l'Ancien Régime à Saint-Domingue aux années de la "République des mulâtres", en passant par l'expédition du Général Leclerc, accompagnée, comme on l'a vu, de son épouse Pauline Bonaparte, pour rétablir dans l'île l'esclavage et par le régime despotique instauré par Henri Christophe. Ti Noel traverse régimes et vicissitudes et c'est lui qui tirera, juste avant de disparaître, la leçon d'une longue suite de misères et de souffrances qui ne se limitent pas à son cas personnel, mais qui concernent "le royaume de ce monde". La formule, empruntée au Nouveau Testament, est, on le voit, fortement laïcisée et radicalisée.

C’est dans une tonalité toute différente qu'apparaît Filomeno, le serviteur noir d'un riche maître créole mexicain. Nous sommes en plein XVIII ème siècle et en route pour Venise où maître et valet vont faire connaissance de Vivaldi, Haendel, Scarlatti et assister à la première représentation de l'opéra Motezuma composé par Vivaldi. Quand Filomeno raconte l'histoire de son illustre aïeul, il réemploie un passage de La Musique à Cuba consacré à la première poésie "cubaine" où apparaît un personnage noir, El Espejo de Paciencia de Silvestre de Balboa. Quand il tombe, en plein Ospedale della Pietá, sur une peinture où est représenté le serpent de la Genèse, l'animal lui inspire aussitôt un "son", poésie chantée et dansée qui figure aussi dans La Musique à Cuba. "Son" est entendu comme "sum", latin d'église, par Vivaldi (Carpentier 1976: 59-60). Le dialogue des cultures, le 
métissage culturel qui définit, selon Carpentier, le baroque, sont exploités ici de manière ludique, parodique et allusive dans un texte qui multiplie les anachronismes. Mais le ton devient plus grave, après la représentation de l’opéra: le maître créole, descendant d'Espagnols, s'est surpris à prendre le parti des Indiens. Mélancolique, il laisse Filomeno à Venise où un frère de race, Louis Armstrong donne un concert. Le prolétaire Filomeno, en allant écouter "le cuivre incomparable" d’Armstrong, réaffirme la valeur essentielle pour lui du rythme.

C'est encore le rythme qui anime, dans La Danse Sacrale, Calixto, un jeune ouvrier maçon qui débarque un beau jour dans l'école de danse que dirige à La Havane Vera, une Russe blanche, comme l'était la mère de Carpentier. Le roman est une longue fresque historique et culturelle qui fait passer de la Guerre d'Espagne et de la défaite de la Révolution au triomphe de celle-ci, à Cuba, en 1959. Calixto devient vite indispensable: administrateur compétent, il se révèle aussi un admirable danseur. Il recrute même de jeunes élèves qui, comme le remarque Vera, ont comme lui le sens inné du rythme. Mais, dès les premières manifestations de l'offensive menée par Fidel Castro contre le régime dictatorial de Batista, Calixto s'engage, sans hésiter, dans la lutte révolutionnaire.

La présentation, volontairement succincte, voire schématique, des quatre romans, éclaire cependant l'image de la trajectoire de vie qui avait été annoncée. Elle la précise en faisant porter l'attention sur la fin, l'issue des romans. Au-delà de différences évidentes entre les textes (ampleur, tonalité), c'est à l'homme noir qu'est dévolue la fonction de conclure, même de façon provisoire, une action qui a été commencée sans lui ou à son insu. L'histoire dans son versant américain abolit le drame qui est survenu en Afrique et en Europe: autre pouvoir, après traversée atlantique (traversée de drames) de l'espace américain.

Trajectoire de vie, mais finalisée. Cette caractéristique ne concerne pas seulement la poétique propre à chaque roman: elle engage l'interprétation qu'on peut en faire. Même dans le cas de Menegildo que l'on suit depuis la naissance jusqu'à la mort, les différentes étapes de sa vie entrent en compétition avec l'évocation d'un faux quotidien dont on détaille le pittoresque et les pratiques magico-religieuses. Il est moins le protagoniste d'une 
histoire, la sienne, qu'un contrepoint dans une action, un argument qui le dépasse, avec la naissance posthume d'un autre Menegildo. Et c'est ici que l'on peut voir comment le roman de Carpentier pose, dès Ekoué-Yamba-Ó, une problématique que Sartre va développer dans Orphée Noir (1947), la préface à la fameuse anthologie poétique de Senghor. Rappelons que l'homme noir, selon Sartre, est à la fois le symbole de l'humanité souffrante, tel qu'il peut lui apparaître dans la tradition du negro spiritual américain, et le protagoniste d'une lutte qui ne sera plus seulement de races, mais de classes. Le parallèle avec Carpentier est évident et éclairant.

La triade de personnages féminins et le quatuor d'hommes noirs ne doivent pas faire oublier qu'ils redisent à leur manière l'expérience première du romancier dont la jeunesse et l'adolescence a oscillé entre deux continents. On comprend, sans sacrifier à la biographie, comment, au long des romans de Carpentier, revient une figure insistante, obsédante: non pas simplement celle du voyageur, mais celle du personnage embarqué, accoudé au bastingage, regardant la mer ou l'océan, dédoublements évidents du romancier. On l'a vu avec Pauline, avec Esteban qui passe de sa Cuba natale à Paris, puis refera le chemin inverse, avec Sofía contemplant les merveilles de la Mer Caraïbe (et refaisant à quelques années d'intervalle le même voyage). On ajoutera, dans cette galerie, Mastaï, futur Pie IX, au début de La Harpe et l'Ombre, songeant à canoniser Colomb, refaisant le voyage atlantique ouvert, historiquement, par Colomb, transformé d'une certaine manière par la traversée atlantique et la découverte de l'espace américain, en l'occurrence chilien. A leurs côtés, d'autres voyageurs ont choisi indifféremment la terre ou la mer et le voyage entre deux continents devient le grand événement de leur vie: les deux voyages du narrateur des Pasos Perdidos, le second corrigeant l'enthousiasme du premier, lui procurant la véritable conscience historique dont a besoin tout homme, surtout lorsqu'il prétend être créateur. C'est Juan de Amberes, simple soldat qui va et vient entre la vieille Europe et la jeune Amérique, suivant le chemin de Saint-Jacques (El Camino de Santiago). C'est enfin le Premier Magistrat, dans El Recurso del Método/Le Recours de la Méthode qui affirme, moment où il quitte l'Atlantique pour entrer dans l'aire caraïbe: "L'air a déjà une odeur différente" (Carpentier 1975: 42). ${ }^{6}$ 
Pour tous ces personnages, l'Atlantique est le lieu de passage, de transformation, espace proprement utopique, ni tout à fait l'Europe et pas encore l'Amérique, mais aussi l'une et l'autre, comme trait d'union. ${ }^{7}$ L'Atlantique va encore jouer ce rôle dans le transfert d'un continent à l'autre de l'esthétique baroque, base de la poétique romanesque de Carpentier.

Pour Alejo Carpentier, le choix d'une esthétique pour élaborer une poétique romanesque, d'abord le "réel merveilleux", puis le baroque, a été au service d'une affirmation identitaire, individuelle et à l'échelle du continent "américain". Cette affirmation s'est exprimée, au plan d'un certain imaginaire, à travers la construction d'espaces qui s'écrivent en fonction de glissements et de superpositions spatiales à évidentes portées poétiques. On ne saurait donc oublier l'opposition qui traverse l'œuvre romanesque, dans son ensemble, entre un "ici" et un "ailleurs" (acá/allá) qui, jusque dans son balancement et sa formulation binaire met en scène l'espace où va s'opérer le choix et le formulation d'une esthétique: l'espace atlantique, l'espace intermédiaire qui sépare et relie les deux ensembles continentaux.

Partons, sans schématisme aucun, de ce qu'on appellera la "thèse" ou les enjeux poétiques qui se posent à Carpentier à son arrivée en Europe, à Paris, au début des années 30: imiter l'esthétique avant-gardiste, le surréalisme, être, comme il l'a souvent noté, un épigone. Or, Carpentier souhaite suivre le mot d'ordre de Simón Rodríguez, précepteur de Simón Bolívar: "Nous devons être originaux"/Tenemos que ser originales. C'est pourquoi, en un second temps (une sorte d'antithèse), il critique le surréalisme (européen) et propose le "réel merveilleux" (américain et d'abord caraïbe, les Antilles étant, depuis la découverte, la antesala del Nuevo Mundo) comme solution à l'écriture de l'espace américain: une naturalisation ou américanisation du surréalisme. Mais cette solution qui a contre elle celle d'être, précisément, une antithèse de l'Europe, va se transformer en proposition esthétique, le "baroque", comme expression profonde du continent américain (hispano-américain). Or, 
le baroque est d'origine européenne: il faudra donc que Carpentier prouve que le baroque est, tout autant que le "réel merveilleux", l'expression d'un ethos américain, ce qu'il va démontrer à la fois dans quelques textes critiques et dans les romans postérieurs au Siècle des Lumières.

“Lo real maravilloso", le réel merveilleux, suscite, on l'a vu, un texte qui n'est ni un manifeste, ni un programme, mais une préface au premier roman reconnu de Carpentier, Le Royaume de ce Monde en 1949. Ce texte est une machine de guerre contre le surréalisme européen, c'est-à-dire en premier lieu français. Cette dimension polémique qui s'affirme dans les premières années de l'après-guerre ne cessera jamais. On la retrouvera encore dans La Danse Sacrale (1978), avant-dernier roman, à la faveur d'un retour aux années 30, celles pour Carpentier de l'expérience parisienne, puis madrilène, au début de la guerre d'Espagne. ${ }^{8}$

Au-delà de la polémique et de la fidélité à l'ami, Robert Desnos, surréaliste dissident, il faut chercher le fondement de ces attaques dans la volonté de ne pas être un simple épigone d'un mouvement européen: le réel merveilleux a été un... merveilleux outil notionnel qui a permis à Carpentier de préciser une identité américaine qu'il cherchait à définir depuis son départ de Cuba en 1928 et pendant la décennie qui a correspondu à son apprentissage poétique à Paris. "Lo real maravilloso" doit à l'évidence beaucoup au "merveilleux" surréaliste du Premier Manifeste et aussi à l'ouvrage de Pierre Mabille, Le Miroir du Merveilleux (1940). Il repose de fait sur un double postulat: l'Amérique, dans sa nature même, offre, à l'état brut, un merveilleux que l'Europe obtient de façon artificielle, intellectuelle. Aussi l'Amérique est-elle, sur ce point au moins, supérieure à l'Europe.

L'idée de "supériorité", fondée sur la comparaison avec une dimension culturelle étrangère (une des orientations majeures sur laquelle s'exerce le comparatisme), sert à la définition d'une identité et à inverser le rapport de force entre Amérique et Europe, entre le Nouveau monde et l'Ancien qui s'est établi depuis le XVIème siècle. Il faut situer la position de Carpentier, sa revendication identitaire, dans le prolongement du mouvement modernista hispano-américain des dernières années du XIX ème siècle. Entendons: l'Amérique cesse de fournir et d'enrichir l'Europe; elle inverse à son profit une situation qui 
n’est plus de dépendance. C’est selon nous le Haïtien René Depestre qui a le mieux compris ce qu'a pu être l'entreprise du "real maravilloso" lorsqu'il affirme:

La notion de réel merveilleux est la négation poétique et romanesque des circonstances historiques de la conquête coloniale. C'est l'antithèse artistique de la realpolitik qui aura été à l'œuvre aux Amériques à partir de l'aventure de Christophe Colomb. C'est son inverse, que j'appelle aussi la realutopie des humiliés et des offensés. (Depestre 1998: 112)

Nous sommes aussi, avec ce jugement, au plus proche d'une problématique que certains nomment "postcolonialisme".

La supériorité américaine qui est plus de l'ordre de la Nature que de la Culture, son dynamisme vital trouvent leur expression romancée dans Los Pasos Perdidos/Le Partage des Eaux, où sont multipliées les piques contre le surréalisme et aussi les allusions (à commencer par le titre qui est un clin d'œil à Breton, ou celles qui concernent Rosario, la femme essentielle, primordiale, amante et muse tout à la fois). Le narrateur anonyme, sorte d'homo americanus, en quête d'identité, à la recherche de sa langue maternelle, l'espagnol, puisqu'il vit dans une ville qui ressemble à New York, évoque l’Europe qui a produit la IXème Symphonie de Beethoven et les horreurs de la Seconde Guerre Mondiale, et qui apparaît, au début des années 50, comme exsangue et ruinée.

De l'esthétique passons à la poétique: le "réel merveilleux" est associé à un genre essentiellement "américain", la chronique, le genre qui a surgi dans le temps même où se faisaient la conquête et la colonisation des immenses territoires reconnus par les Espagnols. On rappellera comment se termine la préface au Reino de este Mundo: "Mais qu'est-ce que l'histoire de l'Amérique tout entière si ce n'est une chronique du réel merveilleux?" (Carpentier 1980: 349) Or cette prise de position va évoluer après 1960, après la publication du Siècle des Lumières. La notion de baroque va se substituer à celle de réel merveilleux pour aboutir en 1975 à la conférence donnée à l'Ateneo de Caracas dans laquelle Carpentier procède à un étonnant renversement de sa propre chronologie en choisissant comme titre: "Lo Barroco y lo Real Maravilloso"/"Le Baroque et le Réel Merveilleux" (Carpentier 1981: 111-135). 
Dans ce long exposé, il part de la théorie de Eugenio d’Ors sur la pérennité et la récurrence du baroque à travers les âges (le eon baroque). Le baroque est un "esprit" et non "un style historique". Sont donc baroques l'Arioste, Shakespeare, Rabelais, Novalis, Rimbaud, Lautréamont (belle captation faite sur le dos du surréalisme!) et... Proust. Comme le dernier nom peut surprendre (et il veut à coup sûr qu'il surprenne), Carpentier cite le passage de La Prisonnière où le narrateur, dans le lit d'Albertine, entend les bruits et les cris de la rue et, "jouant de façon vertigineuse avec le temps" (idem 1981: 122), s'adonne à des parallèles ou à des superpositions phoniques entre le chant grégorien, Pelléas et Mélisande, et "met en relation" le chant du vendeur de mouron pour les petits oiseaux avec le chant médiéval. On appréciera deux opérations "poétiques": parallèle et relation.

Cette approche est essentielle à la démonstration de Carpentier. En mettant en relief le jeu des anachronismes, il peut, sans le dire explicitement, inclure Los Pasos Perdidos dans l'esthétique baroque et illustrer la proposition avec le tout récent Concierto Barroco. Il exploite l'idée, capitale à ses yeux, de la "mise en relation" d'époques différentes, de contenus culturels différents, de surimpressions d'espaces et de temps pour en arriver à cette nouvelle proposition identitaire: “L'Amérique, continent des symbioses, des mutations, des vibrations, des métissages a été baroque depuis toujours" (idem: 123). Le baroque est assimilé à un “mestizaje cultural/métissage culturel” (idem: 126). Et l'on se souviendra que, dans Concert Baroque, le maître mexicain découvre son américanité, sa solidarité avec les anciens Mexicains, après la représentation d'un opéra baroque de Vivaldi sur la conquête du Mexique. Il a beau crier "faux, faux et faux!" durant le spectacle, il se surprend, lui, descendant d'Espagnols, à prendre le parti des Indiens.

Qu'on ne cherche pas dans cette conférence une quelconque nouveauté dans la présentation du réel merveilleux. Carpentier oppose un merveilleux européen "fabriqué" à un merveilleux "latent" dans tout le continent. Il demande aussi qu'on compare la mer du Cimetière Marin de Valéry à l'Orénoque dans le roman Canaima de Rómulo Gallegos pour démontrer ce qu'il ne faut jamais oublier: la débilité de l'ancien monde et la démesure du nouveau. Qu'il s'agisse du réel merveilleux ou du baroque, ces deux esthétiques se caractérisent comme un moyen de définir une essence américaine, en soi et en relation plus 
ou moins affirmée avec l'Europe. Et aussi comme des moyens de dire l'espace américain, de chanter la Nature américaine, puisque Carpentier n'hésitera pas à comparer le roman nouveau dont il se fait le champion à une nouvelle écriture épique, un nouvel epos.

La mise en mots d'un espace, le passage d'un ordre naturel à un ordre culturel représente l'une des bases de l'esthétique de Carpentier. Il importe de dire, d'exposer, d'expliquer des lieux, des bouts de monde, l'espace caraïbe essentiellement, d'en faire l'histoire, de le constituer en savoir, de le rendre habitable, au sens moral et symbolique, d'en faire un monde possible et surtout un monde exemplaire. Il ne s'agit nullement de faire coïncider un espace avec une langue, ce qui est le propre de l'idéologie. Il s'agit, au contraire, de faire que la lettre, par sa force poétique, donne à l'espace, au réel, sens et cohérence. Il importe, pour Carpentier, de faire du romancier un "nouvel Adam" dont la tâche première est de "nommer les choses". Il y a chez Carpentier ce que j'ai pu appeler un "complexe d'Ithaque": rendre "classique", hors du temps, un espace limité, déterminé.

Pour dire le monde ou tel espace particulier, il faut des mots nouveaux: la complexité du réel américain appelle des mots “agglutinés”. C’est ce que pense Esteban dans Le Siècle des Lumières dans le temps même où il découvre "les premiers baroques de la création", "l'univers des symbioses" de la mer caraïbe: "Esteban était rempli d'étonnement quand il remarquait que le langage, en ces îles, avait dû utiliser l'agglutination, l'amalgame verbal et la métaphore, pour traduire l'ambiguïté formelle de choses qui participaient à plusieurs essences" (Carpentier 2005: 240).

La métaphore, on le sait, est l'une des figures de prédilection du baroque. Jean Rousset, dans son Dernier Regard sur le Baroque, rappelle les mots par lesquels Umberto Eco définit le travail métaphorique dans son roman L'Isle du Jour d'Avant: "relever des notions éloignées et trouver des ressemblances en des choses dissemblables" (Eco apud Rousset 1998: 18). Cette proposition recoupe de façon étonnante la définition de la culture selon Carpentier (1981: 17). ${ }^{9}$ Mais il vaut mieux rappeler l'épisode de La Danse Sacrale où la notion de métaphore permet à Enrique d'oublier les doutes qu'il nourrissait à l'égard de la révolution cubaine et de découvrir un sens à son travail et à sa vie: "Il faut travailler métaphoriquement" dit-il: "Figure de rhétorique par laquelle on transporte le sens d'un mot 
sur un autre, au moyen d'une comparaison mentale" (Carpentier 1978: 516). Dans la perspective de notre étude, la métaphore est l'expression même de ces voyages atlantiques, "transatlantiques", de ces transferts de l'ancien au nouveau monde.

La métaphore autorise tous les jeux de rapprochement, comparaison, similitudes, équivalences qui constituent une large part de la poétique de Carpentier, en particulier la poétique de l'espace. Ce qu'il découvre, dès le voyage en Haïti et l'élaboration du Royaume de ce Monde, puis dans Le Partage des Eaux, c'est l'existence de temps dédoublés, juxtaposés, qui apparaissent comme un élément constitutif du continent américain. Les dédoublements ne s'opèrent pas seulement selon l'axe chronologique: les deux parties de l'île, française et espagnole, se superposent, de même que, dans Le Siècle des Lumières, l'espace caraïbe se superpose à l'espace méditerranéen. Dédoublements et superpositions constituent ce que nous avons appelé des "espaces romanesques". Ils justifient tout aussi bien l'idée du baroque comme métissage culturel que le réel merveilleux, dans une certaine proximité avec le surréalisme.

$\mathrm{Au}$ tout début du Royaume de ce Monde, l'esclave noir Ti Noel contemple, en attendant son maître, deux devantures contiguës, celle d'un tripier et celle d'un perruquier, qui offrent à sa méditation (culturelle, politique) des têtes de veau d'une blancheur fascinante et des têtes de cire couronnées de perruques. Il faut admettre, d'entrée de jeu, que l'alliance de ces deux devantures, est moins "laborieuse" (mot de la préface au roman à propos du surréalisme) que toutes celles qui procèdent de la rencontre d'un parapluie et d'une machine à coudre sur une table de dissection (pour reprendre la célèbre comparaison citée dans le même prologue). La scène, quasiment inaugurale, élaborée par Carpentier, est de fait d'inspiration radicalement différente de l'esprit surréaliste. Il s'agit ici de procéder à la mise en scène métaphorique d'une image de la Révolution imminente qui menace les colons de l'île. Cette contiguïté des têtes coupées, de la blancheur et de l'aristocratie portant perruque possède une charge historique, culturelle, éthique même, puisqu'elle est évoquée à travers les yeux d'un esclave noir qui se pose des questions sur son maître et sur la lointaine mère Afrique. La scène est une vision prémonitoire de la Révolution ou, si l'on 
préfère, une métaphore inaugurale, au même titre que la séquence, inaugurale elle aussi, du Siècle des Lumières avec la "Machine" sous sa housse, c'est-à-dire la guillotine.

C'est ainsi que la présence d'un Lenormand de Mezy (personnage historique) précipite l'île d'Haïti dans une métamorphose en Normandie. L'île de la Tortue où s'est réfugiée Pauline Bonaparte devient une Corse seconde, île natale de l'héroïne. C'est, dans Concert Baroque, la lagune de Venise qui fait place à la lagune de Mexico, les Mori qui sonnent les heures deviennent les frères d'Othello, Maure de Venise, et de Filomeno, serviteur noir du maître mexicain. Les trompettes bibliques se changent en trompette de Louis Armstrong. Ainsi se crée un vaste réseau de correspondances qui procèdent du principe poétique de l'analogie, autre recours de la poétique baroque, mais fondement de toute écriture, imagination poétique, comme l'a rappelé Octavio Paz puisqu'en fait il s'agit de voir en une chose... autre chose. ${ }^{10}$ Assurément, métaphore et analogie altèrent plus profondément la perception du réel que l'association surréaliste, l'illumination par le merveilleux de la réalité, le changement du quotidien en insolite.

Ces changements, superpositions de temps et d'espaces, sont comme favorisés par les incessants va-et-vient que font les personnages de Carpentier entre Europe et Amérique, par les redécouvertes qu'ils font de leur "ici", après de longues années d'absence "ailleurs". Il semble qu'ils pourraient prendre à leur compte le fragment du poème nahuatl cité en épigraphe à la IIIème partie de La Danse Sacrale: "Devrai-je être à nouveau semé?" (Carpentier 1978: 185). Mais c'est aussi Carpentier lui-même qui vit et éprouve non sans quelque amusement des analogies entre La Havane et des "coins" d'Europe, au moment même où il retrouve la métropole cubaine après dix ans d'absence.

La re-naissance, la redécouverte de l'espace ne s'accompagne d'aucune remise en cause du réel, aucune subversion. Tout au contraire, il faudrait parler d'une révélation du réel, de sa célébration. Quand Carpentier parle, à propos du roman nouveau américain, de son "devoir de révélation", on comprend à quel point le travail poétique de révélation est la phase qui suit celle qui a consisté à amasser des faits, des lectures (ce qu'il a fait à Paris pendant dix ans) et à les mettre en rapport. En même temps, on mesure comment ce travail poétique semble proche du Surréalisme et lui est pourtant totalement opposé. Relation, 
révélation sont, de façon significative, les deux notions que retiennent Maurice Blanchot et Octavio Paz dans l'hommage rendu à André Breton par la NRF (1967, no 172). Mais il s'agit pour le poète surréaliste d'aller au plus profond du psychisme humain, pour en saisir les aspects encore inédits, inavoués, insolites, pour lever enfin toute censure, et les exprimer, y compris sous forme d'écriture automatique.

Tout autre est le fondement de sa poétique. Très tôt, il a invoqué, en citant Unamuno, le principe selon lequel il importe "de trouver l'universel au cœur du singulier" (Carpentier 1983: 230). Ce principe justifie à lui seul la volonté de Carpentier de dire le monde, de "nommer les choses" et d'être compris par le public le plus large. L'expression poétique nouvelle est inséparable de la reconnaissance la plus large possible. Citant à nouveau Unamuno ("Il faut trouver l'universel au sein même du local"), il définit en 1958 ce qu'est la nouvelle littérature hispano-américaine - et son écriture personnelle - comme une tentative pour "se désexotiser" /"y es que nos vamos desexotizando" (Carpentier 1985: 51).11 Entendons: tourner le dos au réalisme pittoresque et parier pour une dimension sinon universelle, du moins reconnue par le public le plus large possible. Dix ans plus, il parlera de "déprovincialiser"/desprovincializar la littérature en "révélant" (le mot "revelar" est en italique) des "réalités encore inédites" (idem: 164).12 Enfin, en 1975, il terminera son exposé sur le "baroque", déjà cité, par ces mots: "Nous serons les classiques d'un énorme monde baroque qui nous réserve encore, à nous et au monde, les plus extraordinaires surprises" (Carpentier 1981: 135; nous soulignons).

La visée constante de Carpentier a bien été celle de substituer au magistère de l'ancien monde, celui d'un nouveau monde, nourri de l'ancien, mais conçu selon un étonnant renouvellement du principe antique, classique, de l'imitation, à savoir l'émulation. Au travail de sape, en profondeur, en plongée, du surréalisme, Carpentier oppose la relation (mot clé de sa définition de la culture) et la révélation (principe de sa poétique romanesque), la célébration du "royaume de ce monde", horizontale, historique, géographique. Et c'est cette voie poétique tracée par ces mots qui vient donner tout son sens à ce que nous avons souhaité privilégier dans cet article: les traversées atlantiques. 


\section{NOTES}

${ }^{1}$ Nous renvoyons chaque fois que cela est possible aux excellentes traductions françaises de René L.-F. Durand, sans ignorer l'original en espagnol.

${ }^{2}$ Le texte espagnol est plus expressif: "Paulina descubrió que el mar se estaba renovando".

${ }^{3}$ Le texte espagnol dit "los idos".

${ }^{4}$ La citation de Lewis Carroll et cette phrase sert aussi d'épigraphe au roman.

5 Toutefois Carpentier dans ses entretiens avec Ramón Chaó rappelle qu'il n’a rien à renier du message politique qui a pu être exprimé (cf. Chaó, Ramón (1998), Conversaciones con Alejo Carpentier, Madrid, Alianza Ed., 216).

${ }^{6}$ Le texte espagnol dit de façon plus pittoresque et laconique: "Ya huele distinto".

${ }^{7}$ Je reprends Louis Marin ((1970), Utopiques, Paris, Minuit) qui fait de l'utopie l'espace du neutre, ne uter, ni masculin, ni féminin et les deux à la fois.

${ }^{8}$ Voir sur cette période et cette problématique: Pageaux D-H (2007), "Du Surréalisme au Baroque: Regards sur le monde romanesque d'A. Carpentier", in Mesures et Démesure dans les Lettres Françaises au XXème Siècle. Hommage à Henri Béhar, Paris, Champion, 313-326.

${ }^{9}$ Il vient de citer Malraux qui, selon S. de Beauvoir, "quand il voit un chose pense immédiatement à une autre" (Carpentier 1981: 17). Or, selon Carpentier cette "faculté" de penser immédiatement à autre chose est ce qui définit aussi la "véritable culture". La définition est la suivante: "Je dirais que la culture est la masse de connaissances qui permettent à un homme d'établir des relations au-delà du temps et de l'espace, entre deux réalités semblables ou analogues et d'expliquer l'une en fonction de ses similitudes avec l'autre qui a pu se produire bien des siècles auparavant" (idem: 17 ).

10 "Analogie, transparence universelle: en ceci voir cela" (Paz 1972: 147). La citation superbe rejoint celle que Carpentier a donnée de la culture (cf. supra, note 9).

11 Tiré de "Nuestro Tiempo", mars-avril 1958.

12 Interview donnée pour Verde Olivo, La Havane, 2 février 1969. 


\section{Bibliographie}

Carpentier, Alejo (1975), Le Recours de la Méthode, Paris, Gallimard.

-- (1976), Concert Baroque, Paris, Gallimard, «Folio».

-- (1976), Tientos y Diferencias, Buenos Aires, Calicanto.

-- (1978), La Danse Sacrale, Paris, Gallimard.

-- (1980), Le Royaume de ce Monde, Paris, Gallimard, «Folio».

-- (1981), «Lo Barroco y lo Real Maravilloso», in La Novela Latinoamericana en Vísperas de un Nuevo Siglo, México, Siglo XXI, 111-115.

-- (1983), Chroniques, Paris, Gallimard, «Idées».

-- (1985), Entrevistas, La Habana, Letras Cubanas.

-- (1988), Ekoué-Yamba-Ó, Paris, Gallimard.

-- (2005), Le Siècle des Lumières, Paris, Gallimard, «Folio».

Chaó, Ramón (1998), Conversaciones con Alejo Carpentier, Madrid, Alianza Ed.

Depestre, René (1998), Le Métier à Métisser, Paris, Stock.

Marin, Louis (1970), Utopiques, Paris, Minuit.

Pageaux D-H (2007), «Du Surréalisme au Baroque: Regards sur le monde romanesque d'A. Carpentier», in Mesures et Démesure dans les Lettres Françaises au XXème Siècle. Hommage à Henri Béhar, Paris, Champion, 313-326.

Paz, Octavio (1972), Le Singe Grammairien, Lausanne, Skira, «Les Sentiers de la Création». Rousset, J. (1998), Dernier Regard sur le Baroque, Paris, Corti. 
Daniel-Henri Pageaux

Daniel-Henri Pageaux est Professeur émérite à la Sorbonne Nouvelle/Paris III, codirecteur de la Revue de littérature comparée, membre correspondant de la Academia das Ciências de Lisbonne, docteur honoris causa de l'Université d'Enna/Sicile. Derniers ouvrages publiés: Itinéraires comparatistes, Paris, Jean Maisonneuve éd., 2014, 2 vol.; Lectures indiaocéanes, Paris, Jean Maisonneuve éd., 2016; Musas en libertad. Experiencia poética y creación literaria, Madrid, Turpin ed., 2018. 